\title{
The impact of intermediaries on a negotiation: an approach from game theory
}

\author{
Susana López ${ }^{1} \cdot$ Guillermo Owen $^{2} \cdot$ Martha Saboya $^{1}$ (I)
}

Accepted: 15 September 2021 / Published online: 20 October 2021

(c) The Author(s) 2021

\begin{abstract}
Standard approaches to model interaction networks are limited in their capacity to describe the nuances of real communication. We present a game theoretical framework to quantify the effect of intermediaries on the interaction between agents. Inspired by the seminal work Myerson (1977). on cooperative structures in cooperative games, we set the basis for multidimensional network analysis within game theory. More specifically, an extension of the point-arc game Feltkamp and van den Nouwe51 land (1992). is introduced, generalizing the analysis of cooperative games to multigraphs. An efficient algorithm is proposed for the computation of Shapley value of this game. We prove the validity of our approach by applying it to a intermediaries network model. We are able to recover meaningful results on the dependence of the game outcome on the intermediaries network. This work contributes to the optimal design of networks in economic environments and allows the ranking of players in complex networks.
\end{abstract}

Keywords Cooperative game · Communication game - Games on multigraph · Intermediaries

\section{Introduction}

An intermediary is simply defined as someone mediating an interaction between people who are unwilling or unable to communicate directly to reach an agreement. If two intermediaries can equivalently mediate the communication between two agents, they are said to be perfect substitutes. If, on the other hand, several intermediaries are needed for the agents to communicate, each is a perfect complement to all the others. Two agents can therefore be linked in different ways: through a direct communication

\footnotetext{
$凶$ Martha Saboya

martha.saboya@uam.es

1 Economic Analysis: Quantitative Economics, Faculty of Economics and Business Studies of Universidad Autónoma de Madrid, Madrid, Spain

2 Department of Applied Mathematics, Naval Postgraduate School, Monterey, CA, USA
} 
channel without the need of any intermediaries, through one of the many alternative intermediaries, by employing chains of connected intermediaries or even through a combination of the three. The existence, or more precisely co-existence, of different connection pathways between agents leads to a vast catalog of communication channels, each reaching an agreement with a different degree of efficiency. Evaluating the impact of intermediaries in negotiations is the motivation of the present work.

Negotiations in which a group of agents cooperates on a common project with the objective of maximizing the resulting collective gain can be modelled by a cooperative game with transferable utility. However, in common economical and political contexts, restrictions on the communications typically arise, requiring a redefinition of the cooperative game. Myerson (1977) examines such cooperation structures by introducing graphs to model the communication channels between players. In such representation, players are denoted by graph nodes, which are connected only if a direct communication between the two corresponding players can be established. The graph induces a partition on the set of players into one or more subsets that can negotiate effectively which leads to the so-called graph-restricted game. The Myerson value, or the Shapley value of this graph-restricted game, captures the dependence of the game output on the established cooperations between players. Later works on graph-restricted games are reviewed in Borm et al. (1994). To the best of our knowledge, none of the established frameworks to analyze games on networks deals with networks with multiple edges. However communication channels between two agents can be diverse. They could communicate directly in a personal meeting, through telephone communication, by email, by intermediaries and even using more than one channel at a time. And this is precisely one of the main contributions of this work: a model to study games whose communications are represented by means of multigraphs.

In the present work, we propose a model to analyze games on multigraphs. We extend the model of communication game introduced by Feltkamp and van den Nouweland (1992) to the new setting. We then introduce a method to calculate the Shapley value of this new game using the multilinear extension of a game defined by Owen (1972) and modeling the effective work performed by multiple links through probability distributions. Finally, the impact of intermediaries in a negotiation is evaluated by applying the model for games on multigraphs to intermediaries networks of $n$ agents where substitutable and complementary mediation might co-exist within the network. Assuming a network consisting of two nodes, for example, we explicitly show that the Shapley value of a node (agent) increases with the number of substitutable intermediaries, while it decreases in the case of complementary intermediaries. Moreover, we illustrate the applicability of our model to concrete problems. In the context of a market design, for instance, the optimal number of participant agents on the market is typically of concern. We address such issue by determining the number of intermediaries between two nodes that ensures a Shapley value above a given threshold.

This manuscript is organized as follows: In Sect. 2 we introduce some notation and certain preliminary ideas regarding coalitional games, multilinear extensions, and multigraphs. In Sect. 3, we introduce the multigraph-restricted game. The main result (Theorem 1 in this paper) provides a method for calculating the Shapley value of the multigraph restricted game. In Sect. 4, we apply the developed methodology in the context of negotiations through intermediaries. Our main result of this section 
(Theorem 2) allow us to quantify the impact of a given intermediaries network on the outcome of the negotiation. We offer final comments in Sect. 5.

\section{Preliminaries}

It is assumed that a coalitional game with transferable utilities ( $T U$ game) is a pair $(N, v)$ where $N=\{1,2,3, \ldots, n\}$ is the set of players and $v$, the characteristic function, is a map, $v: 2^{N} \rightarrow \mathbb{R}$, with $v(\{\varnothing\})=0$. For each coalition, $S \subseteq N, v(S)$ represents the transferable utility that $S$ can obtain whenever its members cooperate. The Shapley value (1953) of $v$ is given by:

$$
\Phi_{i}(v)=\sum_{S \subseteq N \backslash\{i\}} \frac{s !(n-s-1) !}{n !}(v(S \cup\{i\})-v(S)), i=1, \ldots, n .
$$

We will assume $v$ is non-negative and super-additive, so that

$$
v(S \cup T) \geq v(S)+v(T), \text { if } S \cap T=\{\varnothing\} .
$$

A coalitional game $v$ is simple if its payoffs are either 1 or 0 , i.e. coalitions are either "winning" or "losing".

Myerson (1977) proposed a model to analyse games whenever cooperation between agents is restricted. In this model, a (simple) graph is considered, in addition to the game, where the players (of the game) are the graph nodes. The graph therefore indicates the set of direct communication channels between pairs of players.

In this paper, we tackle a more general problem, since we consider the existence of several alternative communication channels between agents. Given that we model such extended communication scheme by means of multigraphs, some concepts of graph theory will be useful in our development, see for example Bondy and Murty (1976).

\subsection{Multigraphs}

A multigraph $\mathcal{G}$ is an ordered triple $\left(V(\mathcal{G}), L(\mathcal{G}), \psi_{\mathcal{G}}\right)$; where $V(\mathcal{G})$ is the set of vertices, $L(\mathcal{G})$ is the edge set, and $\psi_{\mathcal{G}}$ is an incidence function, that associates each edge of $L(\mathcal{G})$ with an unordered pair of (not necessarily distinct) vertices of $V(\mathcal{G})$. If $e \in L(\mathcal{G})$, and $\psi_{\mathcal{G}}(e)=\{i, j\}$ we say that $i$ and $j$ are adjacent nodes. Set $L_{i j}(\mathcal{G})=$ $\psi_{\mathcal{G}}^{-1}(\{i, j\})$.

A simple graph $\mathcal{G}=(V(\mathcal{G}), L(\mathcal{G}))$ is a graph where the connection between two nodes is carried out through a single link and it has no loops.

A graph $\mathcal{H}$ is a subgraph of $\mathcal{G}$ if $V(\mathcal{H}) \subseteq V(\mathcal{G}), L(\mathcal{H}) \subseteq L(\mathcal{G})$, and $\psi_{\mathcal{H}}$ is the restriction of $\psi_{\mathcal{G}}$ to $L(\mathcal{H})$. Suppose that $V^{\prime}$ is a nonempty subset of $V(\mathcal{G})$. The subgraph of $\mathcal{G}$ whose vertex set is $V^{\prime}$ and whose edge set is the set of those edges of $\mathcal{G}$ that have both ends in $V^{\prime}$ is called the subgraph of $\mathcal{G}$ induced by $V^{\prime}$ and is denoted by $\mathcal{G}\left[V^{\prime}\right]$. 
A $(i, j)$-path in $\mathcal{G}$ is a finite non-null sequence $i_{0} e_{1} i_{1} e_{2}, \ldots, e_{k} i_{k}$, whose terms are alternatively vertices and edges, such that, for $1 \leq r \leq k$, the ends of $e_{r}$ are $i_{r-1}$ and $i_{r}$ and the vertices $i_{0}, i_{1}, \ldots, i_{k}$ as well as the edges $e_{0}, e_{1}, \ldots, e_{k}$ are distinct and $i=i_{0}, j=i_{k}$.

Two vertices $i, j \in V(\mathcal{G})$ are said to be connected in $\mathcal{G}$ if there is a $(i, j)$ - path in $\mathcal{G}$. A multigraph $\mathcal{G}$ is connected if any two vertices $i$ and $j$ are connected in $\mathcal{G}$.

Connection is an equivalence relation on the vertex set $V(\mathcal{G})$. Thus there is a partition of $V(\mathcal{G})$ into non-empty subsets $V_{1}, V_{2}, \ldots, V_{\omega}$ such that two vertices $i$ and $j$ are connected if and only if both $i$ and $j$ belong to the same set $V_{r}$. The subgraphs $\mathcal{G}\left[V_{1}\right], \mathcal{G}\left[V_{2}\right], \ldots, \mathcal{G}\left[V_{\omega}\right]$ are maximal connected subgraphs of $\mathcal{G}$ and $V_{1}, V_{2}, \ldots, V_{\omega}$ are called connected components of $\mathcal{G}$. Similarly, given $S \subseteq V(\mathcal{G})$ and $A \subseteq L(\mathcal{G})$, there is a unique partition of $S$ by $A$ into nonempty subsets $S_{1}, S_{2}, \ldots, S_{\omega}$ such that two vertices $i$ and $j$ are connected by a subset of links in $A$ if and only if both $i$ and $j$ belong to the same set $S_{r}$. We will denote this partition by $S / A$.

Let us now consider a game $(N, v)$ and a multigraph $\mathcal{G}=(N, L, \psi)$, where the players (of the game) are the nodes of the multigraph. Following Feltkamp and van den Nouweland (1992), we define a new game $w$, a multigraph-restricted game, whose players (pseudoplayers) are the players of the game $v$ as well as the links of $L$. Moreover, the value of a coalition $S \cup A(S \subseteq N, A \subseteq L)$ equals the sum of the $v$-values of the connected components of $S / A$. As Myerson, we use the Shapley value $\Phi(w)$ to assess the power of a pseudoplayer in the multigraph-restricted game. How can one simplify the calculation of $\Phi(w)$, given that the number of pseudoplayers of $w$ is in general relatively high? Note, on the one hand, that the structure of connected components generated by the multigraph is the same as the structure generated by its underlying simple graph (a graph having only a link representing multiple links between two nodes). On the other hand, the value of a coalition $S \cup A$ will be always the same, regardless of which link or links are considered in the communication between pairs of players. Intuitively, we can face the game $w$ as it is played in two steps: first a link is chosen to establish the communication, i.e., a (so called) connection game is played between each pair of communicated nodes, then the game on the simple graph is taking into account. Thus we study $w$ as a compound game as it is defined by Owen (1995):

Definition 1 Let $M_{1}, M_{2}, \ldots M_{n}$ be $n$ disjoint nonempty sets of players. Let $v_{1}, v_{2}, \ldots, v_{n}$ be simple games in $(0,1)$ normalization, with player sets $M_{1}, M_{2}, \ldots M_{n}$, respectively. Let $u$ be a non-negative game over the set $N=\{1,2, \ldots, n\}$; then the $u$-composition of $v_{1}, v_{2}, \ldots, v_{n}$, denoted by $w=u\left[v_{1}, v_{2}, \ldots, v_{n}\right]$ is a game with player set $M^{*}=\cup_{j=1}^{n} M_{j}$ and characteristic function

$$
w(S)=u\left(\left\{j \mid v_{j}\left(S \cap M_{j}\right)=1\right\}\right)
$$

for $S \subseteq M^{*}$

Once we get to see our game $w$ as a composite game, a natural way to calculate $\Phi(w)$ is the use of the multilinear extension of a game, which is defined as: 
Definition 2 The multilinear extension (MLE) of a game $(N, v)$ is given by

$$
F\left(x_{1}, x_{2}, \ldots x_{n}\right)=\sum_{S \subseteq N}\left\{\prod_{i \in S} x_{i} \prod_{i \notin S}\left(1-x_{i}\right)\right\} v(S)
$$

and the Shapley value can be obtained by

$$
\Phi_{i}(v)=\int_{0}^{1} \partial_{i} F(t, t, \ldots t) d t
$$

where $\partial_{i} F$ is the ith partial derivative of the function $F$, namely $\frac{\partial F}{\partial x_{i}}$.

If one takes into account that the composition of games corresponds to the composition of their multilinear extensions, one arrives at a first approximation to compute $\Phi(w)$. One can further simplify the calculation if one observes that the players of one of the games in the composition of $w$ are nonsymmetric. In such situations it is natural to use a weighted Shapley value. Let us recall how to calculate it.

Definition 3 Given a game $(N, v)$ and supposing player $i$ 's time of arrival variable, $X_{i}$, have the distribution $G_{i}(t)=\operatorname{Prob}\left\{X_{i} \leq t\right\}$, the weighted Shapley value $Z_{i}(v)$ associated to the probability distributions, i.e. player i's expected marginal contribution, will be given by the Stieltjes integral

$$
Z_{i}(v)=\int_{0}^{1} \partial_{i} F\left(G_{1}(t), \ldots, G_{n}(t)\right) \cdot G_{i}^{\prime}(t) d t,
$$

where $F$ is the multilinear extension for game $v$.

\section{Games on multigraphs}

The seminal work on cooperative games in which the restrictions on cooperation are given by a graph is due to Myerson (1977). He assumed that the nodes in the graph are the players in the game and that each link represents a direct bilateral communication channel. However, to the best of our knowledge, none of the established frameworks to analyze games on networks deals with networks with multiple links. Multiple links may reflect different types of relationships, different communication channels (courier companies) or different intermediaries in a negotiation. Therefore, multidimensional analysis is necessary to distinguish between different types of interactions, or equivalently, to observe interactions from different perspectives.

In this section we provide a framework within which we can discuss a wide class of cooperation structures, those considering TU games in which cooperation is restricted by means of a multigraph, i.e. those cooperation structures where multiple links are allowed in the graph. In the sequel we only consider multigraphs without loops. 

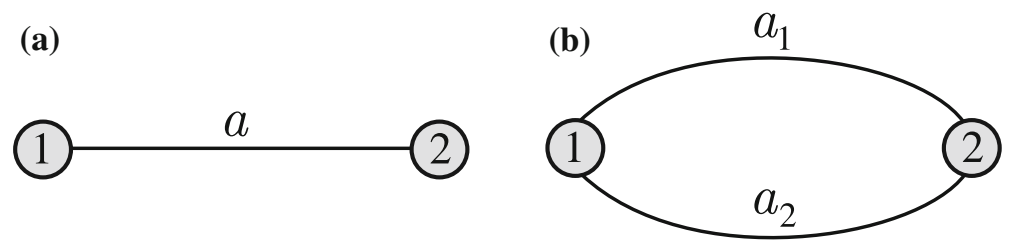

Fig. 1 A simple graph versus a multigraph

Table 1 Point-arc games associated to a simple graph $\left(w_{1}\right)$ and a multigraph $\left(w_{2}\right)$

\begin{tabular}{ll}
\hline$w_{1}$ & $w_{2}$ \\
\hline$w_{1}(\{\varnothing\})=w_{1}(\{a\})=0$ & $w_{2}(\{\varnothing\})=w_{2}(A)=0$ for $A \subseteq L_{2}$ \\
$w_{1}(S)=1$ if $|S \cap N|=1$ & $w_{2}(S)=1$ if $|S \cap N|=1$ \\
$w_{1}(\{1,2\})=v(\{1\})+v(\{2\})=2$ & $w_{2}(\{1,2\})=v(\{1\})+v(\{2\})=2$ \\
$w_{1}(\{1,2, a\})=v(\{1,2\})=4$ & $w_{2}(\{1,2\} \cup A)=v(\{1,2\})=4$ for $A \subseteq L_{2}$ \\
\hline
\end{tabular}

\subsection{Multigraph-restricted games}

Given a game $(N, v)$ and a simple graph $\mathcal{G}=(N, L)$ different types of graph restricted games (communication games) are defined (Borm et al. 1994). The so called point-arc game (Feltkamp and van den Nouweland 1992), which incorporates the players of the game $v$ as well as the links in $L$ as players can be extended in a meaningful way to the new setting, where restrictions in the communication are modeled by a multigraph.

Definition 4 Given a game $(N, v)$ and a multigraph $\mathcal{G}=(N, L, \psi)$, the characteristic function of the associated point-arc game $(N \cup L, w)$ is given by

$$
w(S \cup A)=\sum_{T \in S / A} v(T), \text { for } S \subseteq N, A \subseteq L,
$$

where $S / A$ is the set of connected components of $S$ in $A$.

Note that the "coalitions" in this game are of the form $S \cup A$, where $S \subseteq N$ and $A \subseteq L$. Note also that, if $S$ is connected in $A$, then $w(S \cup A)=v(S)$. We compute the Shapley value $\Phi(w)$ in the usual way.

Example 1 Let $N=\{1,2\}$, with $v(\{1\})=v(\{2\})=1$, and $v(\{1,2\})=4$. Let us consider two different graphs: the simple graph $\mathcal{G}=\left(N, L_{1}\right)$ defined by $L_{1}=\{a\}$ where $a=\{1,2\}$ (Fig. 1a) and a multigraph $\mathcal{G}=\left(N, L_{2}, \psi\right)$, with $L_{2}=\left\{a_{1}, a_{2}\right\}$ where $a_{1} \neq a_{2}$ and $\psi\left(a_{1}\right)=\psi\left(a_{2}\right)=\{1,2\}$ (Fig. 1b).

Let $\left(N \cup L_{1}, w_{1}\right)$ and $\left(N \cup L_{2}, w_{2}\right)$ be the associated point-arc games. Both games take into account that, each player ( 1 or 2) can obtain 1 unit of utility. Together, they can obtain 4 units, but only if they can coordinate. In the absence of this coordination, they obtain only 2 units. Thus, $w_{1}$ and $w_{2}$ are defined by 

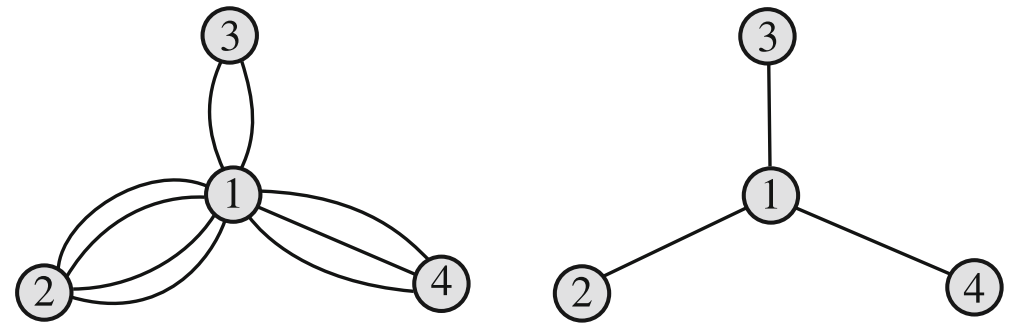

Fig. 2 A multigraph and its underlying simple graph

The Shapley values of $w_{1}$ and $w_{2}$ are then:

$$
\begin{aligned}
& \Phi_{1}\left(w_{1}\right)=\Phi_{2}\left(w_{1}\right)=5 / 3, \Phi_{a}\left(w_{1}\right)=2 / 3 \\
& \Phi_{1}\left(w_{2}\right)=\Phi_{2}\left(w_{2}\right)=11 / 6, \Phi_{a_{1}}\left(w_{2}\right)=\Phi_{a_{2}}\left(w_{2}\right)=1 / 6 .
\end{aligned}
$$

Observe that if $a_{1}$ and $a_{2}$ in $L_{2}$ represent competing messenger services, the Shapley value reveals that competition drives down the two messengers' expectations (compare with Shapley value of $a$ in game $w_{1}$ ).

\subsection{Calculating the Shapley value of game w: the encapsulation procedure.}

Theorem 1 proposes a method of calculating the Shapley value for multigraphrestricted games. The idea is to analyze a game on a multigraph through the game on its underlying simple graph (see Fig. 2).

Definition 5 Given a multigraph $\mathcal{G}=(N, L, \psi)$, the underlying simple graph $\mathcal{G}_{0}=$ $\left(N, L_{0}\right)$ of $\mathcal{G}$ is defined by $a_{i j}=\{i, j\} \in L_{0}$ if and only if $i$ and $j$ are adjacent nodes in $\mathcal{G}\left(L_{i j} \neq\{\varnothing\}\right)$. Set $\left|L_{i j}\right|=l_{i j}$.

Lemma 1 Let $(N, v)$ be a TU game, let $\mathcal{G}=(N, L, \psi)$ be a multigraph and let $\mathcal{G}_{0}=\left(N, L_{0}\right)$ be its underlying simple graph. Let $(N \cup L, w)$ and $\left(N \cup L_{0}, u\right)$ be the corresponding point-arc games. Let $f$ be the multilinear extension of the game u. Then

$$
\Phi_{i}(w)=\int_{0}^{1} \partial_{i} f\left(\vec{t}_{n},\left(1-(1-t)^{l_{l k}}\right)_{a_{l k} \in L_{0}}\right) d t, \text { for } i \in N \text {, }
$$

and for $e \in L_{i j}$

$$
\Phi_{e}(w)=\int_{0}^{1} \partial_{a_{i j}} f\left(\vec{t}_{n},\left(1-(1-t)^{l_{l k}}\right)_{a_{l k} \in L_{0}}\right) \cdot(1-t)^{l_{i j}-1} d t,
$$

Proof We first introduce two families of auxiliary games: for each $i \in N$, the simple 1person game $v_{i}$ is defined by $v_{i}(\{i\})=1$ and for each pair of adjacent nodes $i, j \in N$, 
the connection game $\left(L_{i j}, c_{i j}\right)$ is given by

$$
c_{i j}(A)=\left\{\begin{array}{l}
1, \text { if } i \text { and } j \text { are connected in } \mathcal{G} \text { by elements of } A, \\
0, \text { otherwise, }
\end{array}\right.
$$

for all $A \subseteq L_{i j}$.

Then it is straighforward to show that $w$ can be seen as the compound game $u\left[\left(v_{i}\right)_{i \in N},\left(c_{i j}\right)_{a_{i j} \in L_{0}}\right] .{ }^{1}$ Let $F, f, h_{i}(i=1, \ldots, n)$ and $g_{i} j$ be the multilinear extensions of the games $w, u, v_{i}$ and $c_{i} j$ respectively. Considering that composition of games corresponds to composition of the MLEs, we have:

$$
F(\mathbf{x})=f(h(\mathbf{x})), \text { with } \mathbf{x}=\left(\left(x_{i}\right)_{i \in N},\left(x_{k}\right)_{k \in L}\right),
$$

where

$$
\begin{aligned}
h(\mathbf{x}) & =\left(\left(h_{i}\left(x_{i}\right)\right)_{i \in N},\left(g_{l k}\left(\mathbf{x}_{l k}\right)\right)_{a_{l k} \in L_{0}}\right), \text { with } \mathbf{x}_{l k}=\left(x_{l k}^{1}, \ldots, x_{l k}^{l_{l k}}\right) \text { and } \\
h_{i}\left(x_{i}\right) & =x_{i}, \text { for all } i \in N .
\end{aligned}
$$

Applying the chain rule we get the derivatives of $F$ :

$$
\begin{aligned}
& \partial_{i} F(\mathbf{x})=\partial_{i} f(h(\mathbf{x})) h_{i}^{\prime}\left(x_{i}\right)=\partial_{i} f(h(\mathbf{x})), \text { for } i \in N, \\
& \partial_{e} F(\mathbf{x})=\partial_{a_{i j}} f(h(\mathbf{x})) \frac{\partial g_{i j}\left(\mathbf{x}_{i j}\right)}{\partial e}, \text { for } e \in L_{i j} .
\end{aligned}
$$

Then, applying (1) we have

$$
\Phi_{i}(w)=\int_{0}^{1} \partial_{i} f\left(\vec{t}_{n},\left(g_{l k}\left(\vec{t}_{l_{l k}}\right)\right)_{a_{l k} \in L_{0}}\right) d t, \text { for } i \in N
$$

and

$$
\Phi_{e}(w)=\int_{0}^{1} \partial_{a_{i j}} f\left(\vec{t}_{n},\left(g_{l k}\left(\vec{t}_{l_{l k}}\right)\right)_{a_{l k} \in L_{0}}\right) \frac{\partial g_{i j}\left(\vec{t}_{l_{i j}}\right)}{\partial x_{e}} d t \text {, for } e \in L_{i j}
$$

Finally, we obtain the expressions (3) and (4) for the Shapley value by substituting in the last two expressions $g_{i j}\left(\vec{t}_{l_{i j}}\right)$ by $1-(1-t)^{l_{i j}}$ and $\frac{\partial g_{i j}\left(\vec{t}_{l_{i j}}\right)}{\partial x_{e}}$ by $(1-t)^{l_{i j}-1}$ (see proof of these equivalences in Lemma 4 and Remark 3 in the appendix).

To illustrate the ideas we outlined above, let us examine the next example:

\footnotetext{
${ }^{1}$ The characteristic function of $w$ is then given by

$$
w(S \cup A)=u\left(\left\{j \in N \mid v_{j}(\{j\} \cap S)=1\right\} \cup\left\{a_{i j} \in L_{0} \mid c_{i j}\left(L_{i j} \cap A\right)=1\right\}\right)
$$

for all $S \subseteq N, A \subseteq L$. 


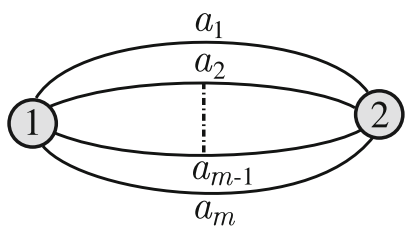

Fig. 3 A multigraph with multiple parallel links

Example 2 Consider the game $(N, v)$ where $N=\{1,2\}$, with $v(\{1\})=v(\{2\})=1$, and $v(\{1,2\})=4$. Consider also a multigraph $\mathcal{G}=(N, L, \psi)$, where $L=$ $\left\{a_{1}, a_{2}, \ldots, a_{m}\right\}$ being $a_{i} \neq a_{j}$ and $\psi\left(a_{1}\right)=\psi\left(a_{2}\right)=\ldots=\psi\left(a_{m}\right)=\{1,2\}$ as represented in Fig. 3.

Let $w$ be the corresponding point-arc game to $v$ and $\mathcal{G}$. We are going to calculate $\Phi_{i}(w)$ and $\Phi_{a_{k}}(w)$ applying last lemma. First note that $w$ can be decomposed as

$$
w=u\left[v_{1}, v_{2}, c_{12}\right],
$$

where $u$ is the point arc-game associated to $v$ and $\mathcal{G}_{0}=\left\{a_{12}\right\}$ (the underlying simple graph of $\mathcal{G}$ ). The MLE $f$ of $u$ is given by

$$
f\left(x_{1}, x_{2}, x_{a_{12}}\right)=x_{1}+x_{2}+2 x_{1} x_{2} x_{a_{12}} .
$$

Now, the derivatives are given by

$$
\begin{aligned}
\partial_{x_{1}} f\left(x_{1}, x_{2}, x_{a_{12}}\right) & =1+2 x_{2} x_{a_{12}}, \\
\partial_{x_{2}} f\left(x_{1}, x_{2}, x_{a_{12}}\right) & =1+2 x_{1} x_{a_{12}}, \\
\partial_{x_{a_{12}}} f\left(x_{1}, x_{2}, x_{a_{12}}\right) & =2 x_{1} x_{2},
\end{aligned}
$$

and applying (3) and (4) we obtain

$$
\begin{gathered}
\Phi_{i}(w)=\int_{0}^{1} 1+2 t\left(1-(1-t)^{m}\right) d t, \text { for } i=1,2, \\
\Phi_{a_{k}}(w)=\int_{0}^{1} 2 t^{2}(1-t)^{m-1} d t, \text { for } k=1,2, \ldots, m .
\end{gathered}
$$

After calculating the integrals we have

$$
\begin{aligned}
\Phi_{i}(w) & =\int_{0}^{1}(1+2 t) d t-\int_{0}^{1} 2 t(1-t)^{m} d t=2-2 \text { beta }(2, m+1) \\
& =2-2 \frac{\Gamma(m+1)}{\Gamma(m+3)}=2\left(1-\frac{1}{(m+2)(m+1)}\right)
\end{aligned}
$$

and

$$
\Phi_{a_{k}}(w)=2 \operatorname{beta}(3, m)=4 \frac{\Gamma(m)}{\Gamma(m+3)}=4 \frac{1}{(m+2)(m+1) m} .
$$


Note that the expressions obtained for the Shapley value, allow us to analyze how $\Phi(w)$ depends on parameter $m$. To advance the simplification of Shapley value and the interpretation of the role of multiple links, let us examine the following example.

As we have seen, the pseudoplayers in the game $u$ are the players in the game $v$ as well as the sets of multiple links between nodes in the multigraph. Clearly, $u$ is an asymmetric game and it is natural to use the weighted Shapley value (2) to evaluate the power of a pseudoplayer in $u$. How then to calculate the distribution of the variable arrival time of a link? This is given by the probability of connection between its pair of adjacent nodes as we can see in following example.

Example 3 Let us consider again the point-arc game $\left(N \cup L_{1}, w_{1}\right)$ associated to game $v$ and graph $\mathcal{G}=\left(N, L_{1}\right)$ of example 1 . This time let us further assume that player $i$ 's time of arrival variable, $X_{i}$ (definition 3 ), have following distribution

$$
G_{1}(t)=G_{2}(t)=t ; G_{a}(t)=1-(1-t)^{2}=2 t-t^{2} .
$$

Then applying (2) we get the value for the game $w_{1}$ (to avoid confusion we call the game $w^{*}$ instead $w_{1}$ along this example)

$$
\begin{aligned}
Z_{1}\left(w^{*}\right) & =\int_{0}^{1} \partial_{1} F\left(G_{1}(t), G_{2}(t), G_{a}(t)\right) \cdot G_{1}^{\prime}(t) d t=\int_{0}^{1}\left(1+2 G_{2}(t) \cdot G_{a}(t)\right) d t \\
& =\int_{0}^{1}\left(1+2 t\left(2 t-t^{2}\right)\right) d t=\frac{11}{6} .
\end{aligned}
$$

Analogously $Z_{2}\left(w^{*}\right)=\frac{11}{6}$. For the link $a$ we have

$$
\begin{aligned}
Z_{a}\left(w^{*}\right) & =\int_{0}^{1} \partial_{a} F\left(G_{1}(t), G_{2}(t), G_{a}(t)\right) \cdot G_{a}^{\prime}(t) d t=2 \int_{0}^{1} G_{1}(t) \cdot G_{2}(t) \cdot G_{a}^{\prime}(t) d t \\
& =2 \int_{0}^{1} t^{2}(2-2 t) d t=\frac{1}{3}
\end{aligned}
$$

Thus

$$
Z\left(w^{*}\right)=\left(\frac{11}{6}, \frac{11}{6}, \frac{1}{3}\right)
$$

Let us compare this value with a result from example 1, there we have

$$
\Phi_{1}\left(w_{2}\right)=\Phi_{2}\left(w_{2}\right)=11 / 6, \quad \Phi_{a_{1}}\left(w_{2}\right)=\Phi_{a_{2}}\left(w_{2}\right)=1 / 6
$$

We see then that the Shapley value for the players coincides with its value in game $w^{*}$ and that

$$
\Phi_{a_{1}}\left(w_{2}\right)+\Phi_{a_{2}}\left(w_{2}\right)=Z_{a}\left(w^{*}\right)
$$


This can be interpreted as the following: the probability distribution of the time of arrival variable of link $a$ represents well the work done by the two different edges in the multigraph (Fig. 1b) from example 1.

In general, we will prove an equivalence between the Shapley value of the game on a multigraph $\mathcal{G}$ and the weighted Shapley value of the game on its underlying simple graph $\mathcal{G}_{0}$, where all the communicative strength of links in $L_{i j}$, described by $g_{i j}$, is attributed to each link $a_{i j}$ of the graph $\mathcal{G}_{0}$ by means of a probability distribution.

We will call encapsulation the process of representing the communicative strength of multiple links between two adjacent nodes $i$ and $j$ through a single link $a_{i j}$, by changing the path of integration or, equivalently, considering the link time-of-arrival distribution the function $G_{a_{i j}}(t)=1-(1-t)^{l_{i j}}$.

Theorem 1 Let $(N, v)$ be a $T U$ game, $\mathcal{G}=(N, L, \psi)$ be a multigraph and $\mathcal{G}_{0}=$ $\left(N, L_{0}\right)$ be the underlying simple graph of $\mathcal{G}$. Let $w$ and $u$ be the corresponding pointarc games. Suppose that the "time of arrival" variable of the elements of $N \cup L$ is uniformly distributed, while the "time of arrival" variable for link $a_{i j} \in L_{0}$ has the following distribution

$$
G_{a_{i j}}(t)=1-(1-t)^{l_{i j}}, \text { for } a_{i j} \in L_{0} .
$$

Then

$$
\begin{aligned}
\Phi_{i}(w) & =Z_{i}(u), \text { for } i \in N, \\
\sum_{e \in L_{i j}} \Phi_{e}(w) & =Z_{a_{i j}}(u), \text { for } a_{i j} \in L_{0},
\end{aligned}
$$

where $Z(u)$ is the weighted Shapley value of $u$ and it is given by (2).

Proof Let us calculate $Z_{i}(u)$ and $Z_{a_{i j}}(u)$ for the point-arc game $u$ associated to $(N, v)$ and the simple graph $\mathcal{G}_{0}$. Let $f$ be the multilinear extension of $u$. Applying (2) we obtain

$$
\begin{aligned}
Z_{i}(u) & =\int_{0}^{1} \partial_{i} f\left(\vec{t}_{n},\left(G_{a_{l k}}(t)\right)_{a_{l k} \in L_{0}}\right) d t \\
& =\int_{0}^{1} \partial_{i} f\left(\vec{t}_{n},\left(\left(1-(1-t)^{l_{l k}}\right)\right)_{a_{l k} \in L_{0}}\right) d t, \text { for } i \in N .
\end{aligned}
$$

Comparing last expression with (3) we can conclude that

$$
\Phi_{i}(w)=Z_{i}(u)
$$

On the other hand, for $a_{i j} \in L_{0}$

$$
Z_{a_{i j}}(u)=\int_{0}^{1} \partial_{a_{i j}} f\left(\vec{t}_{n},\left(G_{a_{l k}}(t)\right)_{a_{l k} \in L_{0}}\right) G_{a_{i j}}^{\prime}(t) d t
$$




$$
=\int_{0}^{1} \partial_{a_{i j}} f\left(\vec{t}_{n},\left(\left(1-(1-t)^{l_{l k}}\right)\right)_{a_{l k} \in L_{0}}\right) \frac{d\left(1-(1-t)^{l_{i j}}\right)}{d t} d t .
$$

Moreover, from (4) we have

$$
\Phi_{e}(w)=\int_{0}^{1} \partial_{a_{i j}} f\left(\vec{t}_{n},\left(\left(1-(1-t)^{l_{l k}}\right)\right)_{a_{l k} \in L_{0}}\right)(1-t)^{l_{i j}-1} d t \text {, for } e \in L_{i j},
$$

then summing over all $e \in L_{i j}$ we obtain

$$
\sum_{e \in L_{i j}} \Phi_{e}(w)=\int_{0}^{1} \partial_{a_{i j}} f\left(\vec{t}_{n},\left(\left(1-(1-t)^{l_{l k}}\right)\right)_{a_{l k} \in L_{0}}\right) \sum_{e \in L_{i j}}(1-t)^{l_{i j}-1} d t
$$

and taking into account that

$$
\sum_{e \in L_{i j}}(1-t)^{l_{i j}-1}=\frac{d\left(1-(1-t)^{l_{l k}}\right)}{d t}
$$

we finally get

$$
\begin{aligned}
\sum_{e \in L_{i j}} \Phi_{e}(w) & =\int_{0}^{1} \partial_{a_{i j}} f\left(\vec{t}_{n},\left(\left(1-(1-t)^{l_{l k}}\right)\right)_{a_{l k} \in L_{0}}\right) \frac{d\left(1-(1-t)^{l_{l k}}\right)}{d t} d t \\
& =Z_{a_{i j}}(u) .
\end{aligned}
$$

In short, the work done by the $m$ parallel links can be "represented" or "modeled" by a single link, by changing the path of integration or equivalently, considering as the link time-of-arrival distribution the function $G_{a_{i j}}(t)=1-(1-t)^{m}$.

Remark 1 In the previous theorem, we have worked with the uniform distribution to describe the time-of-arrival variable of all the players. However, by exploiting the idea behind the multilinear extension, we can go further, we can even model players of a different "nature", simply by assuming that the arrival time variable of each link has a different probability distribution.

\section{Intermediaries networks}

An intermediary is simply defined as someone mediating an interaction between people who are unwilling or unable to communicate directly to reach an agreement. Two agents can be linked in different ways: through a direct communication channel without the need of any intermediaries, through one of the many alternative intermediaries, by employing chains of connected intermediaries or even a combination of the three. The existence, or more precisely coexistence, of different connection pathways between 


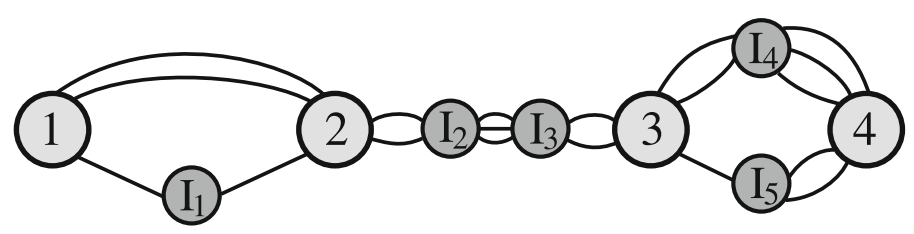

Fig. 4 An intermediaries network

agents leads to a vast catalog of communication channels, each reaching an agreement with a different degree of efficiency.

In Fig. 4 some common situations are represented: agents 1 and 2 can communicate directly through different communication channels or through an intermediary agent; agents 2 and 3 need two intermediary agents to find a compromise and agents 3 and 4 can reach an agreement either through intermediary 4 or through 5 (there may be different communication channels between players and intermediaries).

A network like the one depicted in Fig. 4, and which we will define exactly below, will be called an intermediaries network for the agents $N=\{1,2,3,4\}$. The interests at stake between the agents can be represented by means of a cooperative game $(N, v)$. Given a game $(N, v)$ and an intermediaries network for $N$ and supposing that the intermediaries are given exogenously to the game, in this section we state our main result on intermediaries network-restricted games (Theorem 2), which allow us to compute the Shapley value for those games. To start with we will consider multigraphs $(V, L, \psi)$ with two kinds of set of vertices: $V=N \cup I$, where $N$ is the set of players and $I$ the set of intermediaries.

Definition 6 Let $\mathcal{G}=(N \cup I, L, \psi)$ be a multigraph and $i, j \in N$. A vertex $k \in I$ is an intermediary between $i$ and $j$ if there exist an $(i, j)$ - path such that $k$ is an internal vertex and no element of the path belongs to $N \backslash\{i, j\}$. We denote the set of all intermediaries between $i$ and $j$ as $V_{i j}$. Moreover we shall denote by $E_{i j}$ the set of edges of $\mathcal{G}\left[V_{i j} \cup\{i, j\}\right]$, the subgraph induced by $V_{i j} \cup\{i, j\}$.

Definition 7 Let $\mathcal{G}=(N \cup I, L, \psi)$ be a multigraph. We will say that $\mathcal{G}$ is a network of intermediaries of $N$ if the following two conditions are satisfied:

1. for each vertex $k \in I$ there exist $i, j \in N$ such that $k \in V_{i j}$.

2. $V_{i j} \cap V_{r s}=\varnothing$ for $\{i, j\} \neq\{r, s\}$.

The second condition of the definition above guarantees that connection games are defined on disjoint sets, an essential requirement for the construction of the kind of compound games we have dealt with in this paper. We will denote such a network as $\mathcal{G}_{N}$.

Remark 2 There may be networks whose agents share intermediaries, that is networks where an intermediary agent connects distinct pairs of players, but these kind of intermediaries will not be analyzed in this paper.

Example 4 Let $\mathcal{G}=(N \cup I, L, \psi)$ be the multigraph shown in Fig. 4. We have:

$$
V_{12}=\left\{I_{1}\right\}, \quad V_{23}=\left\{I_{2}, I_{3}\right\}, \quad V_{34}=\left\{I_{4}, I_{5}\right\} .
$$




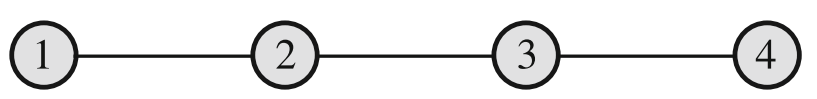

Fig. 5 Underlying simple graph of Fig. 4

The idea now is to "modify" the initial game $(N, v)$ considering all their intermediaries, expanding the definition of multigraph-restricted game to the new context.

Definition 8 Given a game $(N, v)$ and a network of intermediaries of $N, \mathcal{G}_{N}=(N \cup$ $I, L, \psi)$, the intermediaries game $(N \cup I \cup L, w)$ is defined by

$$
w(S \cup J \cup A)=\sum_{T \in(S \cup J) / A} v(T)=\sum_{T \in S / A} v(T), \text { for all } S \subseteq N, J \subseteq I, A \subseteq L,
$$

where $(S \cup J) / A$ is the set of the connected components of $(S \cup J)$ in $A$.

Our main effort, next, is to simplify the calculation of the Shapley value, by using the encapsulation procedure defined in previous section, so that the network in Fig. 4 can be analyzed thought its underlying simple graph represented in Fig. 5.

In order to do so, first, the definition of connection game between two players of $N$ must be modified. Given a game $(N, v)$ and a network of intermediaries of $N$, $(N \cup I, L, \psi)$, the modified connection game $\left(V_{i j} \cup E_{i j}, c_{i j}\right)$ is then defined by

$$
c_{i j}(J \cup A)=\left\{\begin{array}{l}
1, \text { if } i \text { and } j \text { are connected in } \mathcal{G} \text { by elements of } J \cup A, \\
0, \text { else, }
\end{array}\right.
$$

for all $J \subseteq V_{i j}, A \subseteq E_{i j}$.

The next theorem gives the way to calculate the Shapley value of the players of the initial game $(N, v)$ taking into account any intermediaries network.

Theorem 2 Let $v$ a game with player set $N=\{1,2, \ldots, n\}$. Consider two different networks of $N$ : the first one is an intermediaries network $\mathcal{G}_{N}=(V, L, \psi)$ where $V=N \cup\left(\cup_{i, j \in N} V_{i j}\right)$ and $L=\cup_{i, j \in N} E_{i j}$ whereas the second one is the simple graph $\mathcal{G}_{0}\left(N, L_{0}\right)$ where $\{i, j\} \in L_{0}$ if and only if $V_{i j} \cup E_{i j} \neq \varnothing$. Let $w$ and $u$ be the corresponding point-arc games. Suppose that the "time of arrival" variable of the elements of $V \cup L$ is uniformly distributed, while the "time of arrival" variable of link $a_{i j} \in L_{0}$ has the following distribution

$$
G_{a_{i j}}(t)=g_{i j}\left(\mathbf{t}_{\left|V_{i j} \cup E_{i j}\right|}\right)
$$

where $g_{i j}$ is the multilinear extension of the connection game $\left(V_{i j} \cup E_{i j}, c_{i j}\right)$. Then

$$
\begin{aligned}
\Phi_{i}(w) & =Z_{i}(u), \text { for } i \in N, \\
\sum_{k \in V_{i j} \cup E_{i j}} \Phi_{k}(w) & =Z_{a_{i j}}(u), \text { for } a_{i j} \in L_{0},
\end{aligned}
$$

where $Z(u)$ is the weighted Shapley value of game $u$. 


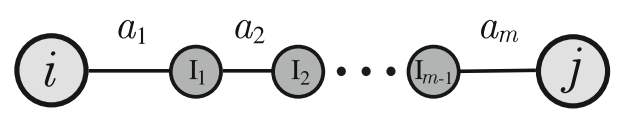

Fig. 6 Chain of connected intermediaries between two adjacent nodes

We omit the proof of Theorem 2. We point out merely that this proof is similar to- - but lengthier than - the proof of Theorem 1. There are two key ideas: the first one is that $w$ can be represented as a compound game as follows:

$$
w=u\left[\left(v_{i}\right)_{i \in N},\left(c_{i j}\right)_{a_{i j} \in L_{0}}\right] \text {, }
$$

where $v_{i}$ is the simple 1-person game defined by $v_{i}(\{i\})=1$ and $c_{i j}$ is the connection game $\left(V_{i j} \cup E_{i j}, c_{i j}\right)$. The fact that the games have disjoint carriers is due to the second condition of definition 7 . The second key idea is that the work done by intermediaries can be represented by probability distributions, for which it is essential to know the multilinear extension of each connection game. Let us then explain how to calculate the multilinear extension $g_{i j}$ of many connection games $c_{i j}$. We start with two basic networks between two players: the network consisting in $m$ parallel links (Fig. 3) and the network formed by $m$ interrelated intermediaries (Fig. 6). In the case of $m$ parallel links we have found that the multilinear extension is $g_{i j}\left(\vec{t}_{l i j}\right)=1-(1-t)^{l_{i j}}$ (Lemma 4). We calculate below the multilinear extension for the case where there are $m$ interrelated intermediaries.

Lemma 2 Let $\mathcal{G}_{N}=(N \cup I, L, \psi)$ be a network of intermediaries of $N$ and $i, j \in N$ two adjacent nodes. Suppose that there is one and only one $(i, j)-$ path (Fig. 6)

Let also $g_{i j}$ be the multilinear extension of the connection game $\left(V_{i j} \cup E_{i j}, c_{i j}\right)$. Then

$$
g_{i j}\left(\vec{t}_{2\left|V_{i j}\right|+1}\right)=t^{2\left|V_{i j}\right|+1}
$$

where $t \in[0,1]$.

Proof The multilinear extension of the connection game $c_{i j}$ is given by

$$
g_{i j}\left(x_{1}, x_{2}, \ldots, x_{2}\left|V_{i j}\right|+1\right)=\prod_{k \in V_{i j} \cup E_{i j}} x_{k},
$$

then

$$
g_{i j}\left(\vec{t}_{2\left|V_{i j}\right|+1}\right)=t^{2\left|V_{i j}\right|+1}
$$

Once it is understood how to deal with these two cases ( $m$ parallel links and $m$ interrelated intermediaries), the multilinear extension of the game $c_{i j}$ determined by many networks of intermediaries is obtained by applying the following results. It is a 


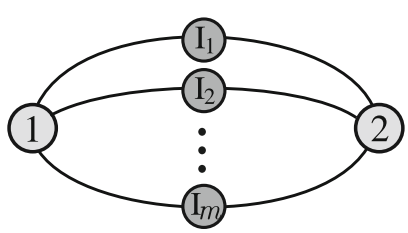

Fig. 7 Alternative intermediaries between two adjacent nodes

matter of applying successive reductions to the set of links and intermediaries in such a way as to simplify the network by appropriately combining the two basic results. This again can be modelled using compound games for which their multilinear extensions is achieved as a composition of multilinear extensions of simpler games.

Lemma 3 Let $\mathcal{G}_{N}=(N \cup I, L, \psi)$ be a network of intermediaries of $N=\{1,2\}$ such that the intersection between every two $(1,2)$-paths is empty. Let $\mathcal{G}^{P}=\left(N, L_{P}\right)$ be the multigraph, where each link $e_{i} \in L_{P}$ represents a $(1,2)-$ path in $\mathcal{G}_{N}$. The connection game $c_{12}$ can be represented as a compound game as follows

$$
c_{12}=c_{12}^{P}\left[\left(c_{(1,2)-p a t h}^{r}\right)_{r \in P}\right] \text {, with } P=\mid(1,2)-\text { paths } \mid,
$$

where $c_{12}^{P}$ is the connection game defined on multigraph $\mathcal{G}^{P}$ and $c_{(1,2)-\text { path }}^{r}$ is a connection game defined on the elements of each $(1,2)$-path. Moreover, if $g_{12}, f$ and $h$ are the multilinear extensions of games $c_{12}, c_{12}^{P}$ and $c_{(1,2)-\text { path }}^{r}$ respectively, we have that

$$
g_{12}\left(\vec{x}_{|I \cup L|}\right)=f(h(\mathbf{x})) \text {, with } \mathbf{x}=\left(\left(x_{i}\right)_{i \in I},\left(x_{k}\right)_{k \in L}\right),
$$

being

$$
h(\mathbf{x})=\left(\left(h_{12}^{r}\left(\mathbf{x}_{s}\right)\right)_{r \in P}\right), \text { with } \vec{x}_{s}=\left(x_{1}^{r}, \ldots, x_{s}^{r}\right)
$$

where $s$ is the number of elements in the corresponding $(1,2)-$ path.

We omit the proof of lemma 3, which relays on the fact that composition of games corresponds to composition of their multilinear extensions. Through next examples we will show how to apply the last lemma.

Example 5 Suppose that the intermediaries network of $N=\{1,2\}$ consists in $m$ alternative intermediaries between 1 and 2 (Fig. 7).

To calculate the multilinear extension of game $c_{12}$, we first calculate the multilinear extension of each path connection game, which in this case is the same for all $m$ paths and applying the lemma 2 is $h_{12}^{r}(t, t, t)=t^{3}$ for $r=1, \ldots, m$. Then the multilinear extension of $c_{12}$ is given by

$$
g_{12}(\vec{t})=f\left(h_{12}^{1}(t, t, t), \ldots, h_{12}^{m}(t, t, t)\right)=f\left(t^{3}, t^{3}, t^{3}\right)=1-\left(1-t^{3}\right)^{m}
$$

where $f(t, t, t)=1-(1-t)^{m}$. 
Example 6 Consider the intermediaries network represented in Fig. 4. To see what happens between agents 1 and 2, first we observe that there are 3 paths connecting 1 and 2 , the multilinear extension of the path connection game going through intermediary 1 is $h_{12}(t, t, t)=t^{3}$, then

$$
g_{12}(\vec{t})=f\left(h_{12}^{1}(t), h_{12}^{2}(t), h_{12}^{3}(t, t, t)\right)=f\left(t, t, t^{3}\right)=1-(1-t)^{2}\left(1-t^{3}\right) .
$$

To calculate the multilinear extension of many connection games you would also need a result in which the pieces we reduce first are the multiple links that are part of a chain of interrelated intermediaries. In any case we would also have a composition but now the roles of the games in the compound game of the previous lemma would be reversed. Let us see how it works with the following example.

Example 7 Consider again the intermediaries network represented in Fig. 4. Note that between agents 2 and 3 there is a chain of interrelated intermediaries and that there are multiple channels between the intermediaries. First we reduce the multiple links and then we apply the result of lemma 3:

$$
g_{23}(\vec{t})=h_{23}\left(g_{2 I_{2}}^{P}(t, t), t, g_{I_{2} I_{3}}^{P}(t, t, t), t, g_{I_{3} 3}^{P}(t, t)\right),
$$

where

$$
g_{2 I_{2}}^{P}(t, t)=1-(1-t)^{2}, g_{I_{2} I_{3}}^{P}(t, t, t)=1-(1-t)^{3}, g_{I_{3} 3}^{P}(t, t)=1-(1-t)^{2} .
$$

Substituting in (6) and applying $h_{23}$ we obtain

$$
g_{23}(\vec{t})=t^{2}\left(1-(1-t)^{2}\right)^{2}\left(\left(1-(1-t)^{3}\right)=t^{5}(t-2)^{2}\left(t^{2}-3 t+3\right)\right.
$$

\subsection{Properties of intermediaries}

A model about intermediaries in a game should be calibrated with the following two properties: the Shapley value of the players increases with the number of alternative intermediaries (agents' negotiation power is greater than having only one intermediary), while it decreases as the number of complementary intermediaries (a large number of intermediaries in series decreases the agents' negotiation power). This follows immediately from the corollaries 1 and 2 .

Corollary 1 Let $N=\{1,2\}, v=u_{12}$ and $\mathcal{G}_{N}=(N \cup I, L, \psi)$ be the network with $m$ alternative intermediaries (Fig. 7). Then

$$
\Phi_{1}(w)=\Phi_{2}(w)=\int_{0}^{1} t\left(1-\left(1-t^{3}\right)^{m}\right) d t=\frac{1}{2}-\left(\frac{1}{3} \frac{\Gamma(m+1)}{\Gamma\left(m+\frac{5}{3}\right)} \Gamma\left(\frac{2}{3}\right)\right)
$$

where $w$ is the corresponding point-arc game to $u_{12}$ and $\mathcal{G}_{N}$. 


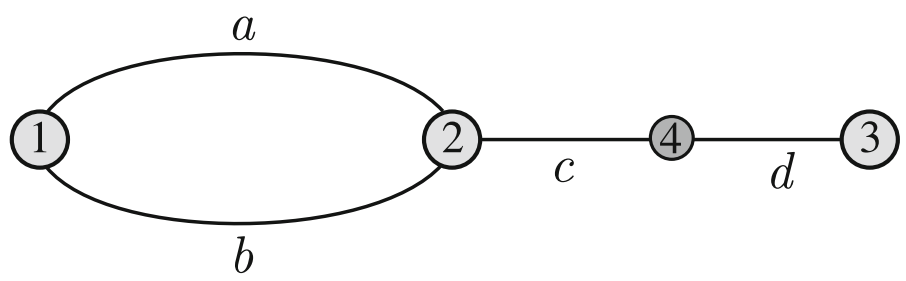

Fig. 8 Diagram of multiigraph $\mathcal{G}_{N}$ of example 8

Corollary 2 Let $N=\{1,2\}, v=u_{12}$ and let $\mathcal{G}_{N}=(N \cup I, L, \psi)$ be the network of $m-1$ intermediaries of $N$ be a chain where players 1 and 2 are the end nodes depicted by Fig. 6. Then

$$
\Phi_{1}(w)=\Phi_{2}(w)=\int_{0}^{1} t \cdot t^{2 m-1} d t=\int_{0}^{1} t^{2 m} d t=\frac{1}{2 m+1},
$$

where $w$ is the corresponding point arc-game to $u_{12}$ and $\mathcal{G}_{N}$.

\subsection{The impact of the intermediaries}

In the context of a market design, for instance, the optimal number of participant agents on the market is typically of concern. We address such issue by determining the number of intermediaries between two nodes that ensures a Shapley value above a given threshold.

\subsubsection{Optimal design of networks in an economic environment}

In the process of defining the intermediaries or the different communication channels between two nodes proposed in this paper, the role of the game $v$ is essential. The characteristic function, $v$, measures the actors economic possibilities. In this way, $v(S)$ represents the profit of $s$ players when there are no restricted relations. We modify the economic position of coalition $S$ so as to reflect the environmental restrictions introduced by the intermediaries network and therefore we are able to optimally design networks in an economic environment. We can solve problems such as: What is the maximum number of interrelated intermediaries between each two players that allows players to retain a given proportion $\alpha$ of their Shapley value in the game? This question can be easily answered by solving following equation: $Z_{i}(u)=\alpha \Phi_{i}(w)$. More insight on how both intermediaries and possible communication channels between nodes affect the results of a game, can be obtained through the following numerical example.

Example 8 Let $N=\{1,2,3\}$ be the set of players, $I=\{4\}$ the set of intermediaries and $\mathcal{G}_{N}=(N \cup I, L, \psi)$ an intermediaries network of $N$ (Fig. 8), where $L=\{a, b, c, d\}$ and $\psi(a)=\psi(b)=\{1,2\}, \psi(c)=\{2,4\}$ and $\psi(d)=\{3,4\}$.

Consider the voting game $v=u_{\{1,2\}}+u_{\{2,3\}}-u_{\{1,2,3\}}$, (where $u_{S}$ is the unanimity game), where $N$ may be considered as a committee of three members, of whom 2 is a 


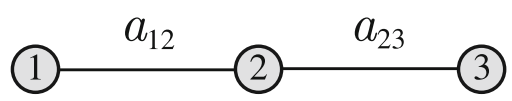

Fig. 9 Underlying simple graph of multigraph in 8

veto-holding chairman. Applying this last theorem, we can obtain the Shapley value of $w$, the intermediaries game to $v$ and $\mathcal{G}_{N}$, by considering the game $u$ corresponding to $v$ and $\mathcal{G}_{N_{0}}=\left(N, L_{0}\right)$ where $L_{0}=\left\{a_{12}, a_{23}\right\}$ (Fig. 8).

The distributions of the "time of arrival" variable for the arcs $a_{12}$ and $a_{23}$ are given by

$$
\begin{aligned}
& G_{a_{12}}(t)=1-(1-t)^{2} \text { and } \\
& G_{a_{23}}(t)=t^{3} .
\end{aligned}
$$

The multilinear extension $f$ of game $u$ is

$$
f\left(x_{1}, x_{2}, x_{3}, a_{12}, a_{23}\right)=x_{2}\left(a_{12} x_{1}+a_{23} x_{3}-a_{12} a_{23} x_{1} x_{3}\right) .
$$

Then

$$
\Phi_{1}(w)=\int_{0}^{1} \partial_{1} f\left(t, t, t, 1-(1-t)^{2}, t^{3}\right) d t=\frac{43}{168}
$$

In a similar way we obtain

$$
\Phi_{2}(w)=\frac{383}{840} \text { and } \Phi_{3}(w)=\frac{11}{280} .
$$

The results are intuitive. The symmetry given by the game between player 1 and player 3 is broken in favor of player 1 because clearly their connections are better than those of player 3. We cannot calculate the Myerson value of this network game, since Myerson only considers simple links. However, the Myerson value of the closest situation (the graph would correspond to graph $\left.\mathcal{G}_{N_{0}}=\left(N, L_{0}\right)\right)$ would be $\mu(v)=\left(\frac{1}{6}, \frac{2}{3}, \frac{1}{6}\right)$, which in this case also coincides with the Shapley value of the game $v$. Clearly $\mu(v)$ do not capture the asymmetry of the link connections. Thus, our treatment of the situation allows us to analyze successfully this kind of asymmetries.

\subsubsection{Times of negotiation}

Another application of the theorem is analyzing the impact of the network of intermediaries on the economic performance of a coalition, taking into account the times of the negotiation. Working with probability distributions allows the following parametric analysis.

Example 9 Consider the network of $N=\{1,2\}$ consisting in $m$ parallel links. The mean of the probability distribution $G_{a}(t)=1-(1-t)^{m}$ which encapsulates the 


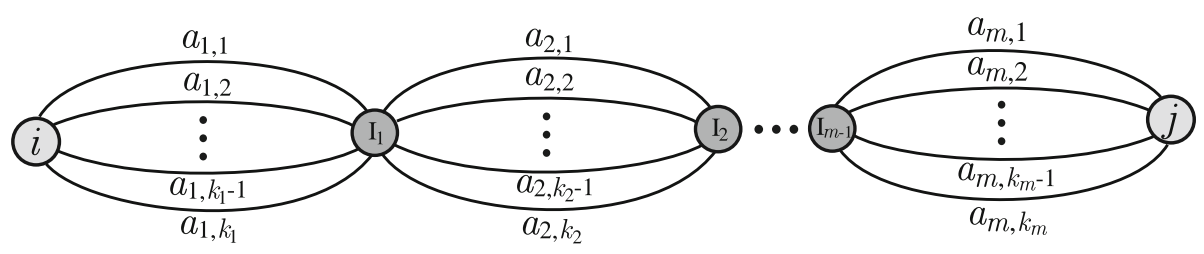

Fig. 10 Diagram of multigraph $\mathcal{G}_{N}$ of example 10

work of the different communication channels is given by

$$
E[X]=1-\int_{0}^{1} 1-(1-t)^{m} d t=\operatorname{beta}(1, m+1)=\frac{1}{m+1} .
$$

Thus

$$
\lim _{m \rightarrow \infty} E[X]=0
$$

Then the average time of arrival of the information through multiple parallel channels decreases as the number of channels increases. In this situation we see that the more the channels are, the smaller is the average time of arrival of the information through the channels. Expressing differently this idea: the more channels the more reliable the network will be.

Example 10 Consider $m-1$ intermediaries interrelated for $N=\{1,2\}$. The mean of the probability distribution $G_{a}(t)=t^{2 m-1}$ which encapsulates the work of the intermediaries is given by

$$
E[X]=1-\int_{0}^{1} t^{2 m-1} d t=1-\frac{1}{2 m}=\frac{2 m-1}{2 m} .
$$

Thus

$$
\lim _{m \rightarrow \infty} E[X]=1
$$

Once again we see that the model reflects well the essence of the work done by intermediaries. In this case, the average time of arrival of the information through the channels increases as the chain of intermediaries is made longer, or in an equivalent way, it is shown that the more intermediaries the less reliable the network will be.

Example 11 Consider $v$ a game, whose player set is $N=\{1,2\}$ and let $\mathcal{G}_{N}$ be the network of intermediaries represented in following figure, being $k_{i}=k$ for all $i=$ $1, \ldots, m$.

Then the probability distribution encapsulating the work of the network is

$$
G_{a}(t)=t^{m-1}\left(1-(1-t)^{k}\right)^{m}, \quad 0 \leq t \leq 1 .
$$


The mean of the distribution $G_{a}(t)$ is given by

$$
\begin{aligned}
E[X] & =1-\int_{0}^{1} t^{m-1}\left(1-(1-t)^{k}\right)^{m} d t \\
& =1-\sum_{j=0}^{m}(-1)^{j}\left(\begin{array}{c}
m \\
j
\end{array}\right) \text { beta }(m, k j+1) .
\end{aligned}
$$

Note that for $j \geq 1$

$$
\left(\begin{array}{c}
m \\
j
\end{array}\right)=\frac{m !}{j !(m-j) !}=\frac{1}{j} \frac{m !}{(j-1) !(m-j) !}=\frac{1}{j} \frac{1}{\operatorname{beta}(j, m-j+1)} .
$$

Finally we get following expression of $E[X]$

$$
E[X]=1-\frac{1}{m}-\sum_{j=1}^{m}(-1)^{j} \frac{1}{j} \frac{\operatorname{beta}(m, k j+1)}{\operatorname{beta}(j, m-j+1)} .
$$

As can be seen, the average time of arrival decreases with communication channels but increases with intermediaries.

\section{Final comments}

In this manuscript we propose a novel approach to compute Shapley values for games in which players are represented in graphs that describe several possible communication channels. Unlike the basic Myerson model, the graph is allowed to have multiple links between the same two nodes, and it is allowed to have nodes that do not correspond to players.

This work represents a methodological contribution to the analysis of games in multigraph settings. As such, it could be employed to study centrality measures in multigraphs, as it has been previously developed for simple graphs in the work of Gómez et al. (2003).

Moreover, this method represents a powerful tool in the growing field of networks economies, where the Shapley value plays a key role in modeling decision making.

Funding Open Access funding provided thanks to the CRUE-CSIC agreement with Springer Nature.

Open Access This article is licensed under a Creative Commons Attribution 4.0 International License, which permits use, sharing, adaptation, distribution and reproduction in any medium or format, as long as you give appropriate credit to the original author(s) and the source, provide a link to the Creative Commons licence, and indicate if changes were made. The images or other third party material in this article are included in the article's Creative Commons licence, unless indicated otherwise in a credit line to the material. If material is not included in the article's Creative Commons licence and your intended use is not permitted by statutory regulation or exceeds the permitted use, you will need to obtain permission directly from the copyright holder. To view a copy of this licence, visit http://creativecommons.org/licenses/by/4.0/. 


\section{Appendix}

Lemma 4 Let $\mathcal{G}=(N, L, \psi)$ be a multigraph and $i, j \in N$ two adjacent nodes. Let also $g_{i j}$ be the multilinear extension of the connection game $\left(L_{i j}, c_{i j}\right)$. Then

$$
g_{i j}\left(\vec{t}_{l_{i j}}\right)=1-(1-t)^{l_{i j}}
$$

where $\vec{t}_{l_{i j}}=\underbrace{(t, t, \ldots, t)}_{l_{i j} \text { times }}$.

Proof By definition 2 the multilinear extension of the connection game $c_{i j}$ is given by

$$
\begin{gathered}
g_{i j}\left(x_{1}, x_{2}, \ldots, x_{l_{i j}}\right)=\sum_{\substack{A \subseteq L_{i j} \\
A \neq \varnothing}} \prod_{k \in A} x_{k} \prod_{k \notin A}\left(1-x_{k}\right) c_{i j}(A) \\
=\sum_{\substack{A \subseteq L_{i j} \\
A \neq \varnothing}} \prod_{k \in A} x_{k} \prod_{k \notin A}\left(1-x_{k}\right) .
\end{gathered}
$$

Then

$$
\begin{aligned}
g_{i j}\left(\vec{t}_{l_{i j}}\right) & =\sum_{k=1}^{l_{i j}}\left(\begin{array}{c}
l_{i j} \\
k
\end{array}\right) t^{k}(1-t)^{l_{i j}-k} \\
& =\sum_{k=0}^{l_{i j}}\left(\begin{array}{c}
l_{i j} \\
k
\end{array}\right) t^{k}(1-t)^{l_{i j}-k}-(1-t)^{l_{i j}} \\
& =(t+(1-t))^{l_{i j}}-(1-t)^{l_{i j}}=1-(1-t)^{l_{i j}} . \\
F\left(x_{1}, x_{2}, \ldots x_{n}\right)= & \sum_{S \subseteq N}\left\{\prod_{i \in S} x_{i} \prod_{i \notin S}\left(1-x_{i}\right)\right\} v(S),
\end{aligned}
$$

Remark 3 The expression (7) can be rewritten as $1-\prod_{k \in L_{i j}}\left(1-x_{k}\right)$. Therefore $\frac{\partial g_{i j}\left(\mathbf{x}_{i j}\right)}{\partial x_{e}}=\prod_{\substack{k \in L_{i j} \\ k \neq e}}\left(1-x_{k}\right)$ and $\frac{\partial g_{i j}\left(\vec{t}_{l_{i j}}\right)}{\partial x_{e}}=(1-t)^{l_{i j}}$.

\section{References}

Bondy JA, Murty USR (1976) Graph theory with applications. The Macmillan Press Ltd., London Borm P, van den Nouweland A, Tijs S (1994) Cooperation and communication restrictions: a survey. In: Gilles RP, Ruys PHM (eds) Imperfections and behavior in economic organizations. Theory and Decision Library (Series C: Game Theory, Mathematical Programming and Operations Research), vol 11. Springer, Dordrecht, pp 195-227 
Feltkamp V, van den Nouweland A (1992) Controlled communication networks. Cahiers du CERO 34:199_ 210

Gómez D, González-Arangüena E, Manuel C, Owen G, del Pozo M, Tejada J (2003) Centrality and power in social networks: a game theoretic approach. Math Soc Sci 46:27-54

Myerson R (1977) Graphs and cooperation in games. Math Oper Res 2:225-229

Owen G (1972) Multilinear extensions of games. Manag Sci 64-79

Owen G (1995) Game theory, III. Academic Press, San Diego

Shapley LS (1953) A value for $n$-Person games. In: Kuhn H, Tucker A (eds) Annals of Mathematical Studies 28, Contributions to the Theory of Games II, pp 307-317

Publisher's Note Springer Nature remains neutral with regard to jurisdictional claims in published maps and institutional affiliations. 